\title{
The role of recorded and verbal information in health information systems: A case study of the Expanded Program on Immunization in Mozambique
}

\author{
Jagrati V. Jani ${ }^{1,2}$, Ilesh V. Jani ${ }^{1}$, Jorge Barreto ${ }^{1}$, Sundeep Sahay ${ }^{3}$
}

\begin{abstract}
Background: There is ongoing interest in strengthening the informational component of the EPI as a mean to enhance the efficacy of service delivery. As developing country governments make significant investments in strengthening health information systems, benefits obtained from these initiatives tend to be below their potential. To improve this, it is argued that such systems need to take into account both formal (documented) and informal forms of information.

Objective: This study measures the accuracy of people-based information with information recorded in the "Road to Health Card".

Methods: 840 mothers were screened in a cross sectional study. Information about measles immunization status as documented in the Road to Health card was compared to mothers' verbal information. The pattern of these answers among mothers presenting the card was compared with those among mothers without the card.

Results: Measles vaccine information was $95.7 \%$ sensitive and $80.0 \%$ specific. Measles vaccination positive answer was $10.0 \%$ higher among mothers without the card. In the sample of mothers without the vaccination card, only $2.0 \%$ never received a card, $53.0 \%$ had forgotten to bring it, and the remaining $44.0 \%$ had lost it.

Conclusion: A key implication of these findings is the need to develop a mother-specific information system that is based at the facility, and an integration of the verbal and tacit information sources while designing the information system. Further recommendation concerns the need to have more targeted information and education programs about the importance of the Road to Health Cards, especially focused for less educated mothers. [Ethiop.J.Health Dev. 2007;21(2):136-141]
\end{abstract}

\section{Introduction}

Immunization has proven to be a low cost and effective intervention to decrease infectious diseases (1). However, vaccine-preventable diseases continue to kill a large number of children each year. The Expanded Program on Immunization (EPI) aims to provide vaccinations in order to decrease the risk of deaths and disabilities from such diseases and thereby contribute to a better quality of life for children living in developing countries. EPI managers use such indicators as vaccine coverage and vaccine efficacy to monitor the performance of EPI and determine the expected outcome of disease incidence and mortality (2). The collection, registration, aggregation, analysis and dissemination of these indicators from one level in the health system to another are fundamentally based on functioning Health Information Systems (HIS).

The need for strengthening HIS in developing countries has now found voice through various means including national policy statements, strategies of international funding agencies, and informatics and public health researchers. Strengthening of HIS is now recognized to be a fundamental requirement for the functioning of the overall health system, including the EPI (3). For instance, the government of Ecuador, through the funding of the International Development Agency, initiated an effort to decentralize and modernize their HIS in the districts to strengthen primary health care service delivery (4). Similarly, there are also ongoing attempts to strengthen HIS in different countries such as Ethiopia, Mozambique, India, Zambia, Nigeria, Cameron and Tanzania $(5,6)$.

In developing countries, a significant amount of the data required for HIS, including data required for the EPI, are typically paper-based,. Some of the required data are obtained from informal sources such as interviews with health service staff and mothers seeking immunization services for their children. This large amount of rather informal and tacit information and knowledge is overlooked as many developing countries introduce Information Communication Technologies (ICTs) to automate their health systems. The role of technology is being considered central in HIS efforts to integrate data collection, processing, reporting and use of information (7). However, often the primary focus of these efforts is on the technology itself, without adequate consideration of the more informal, yet informative, manual or paper based systems which are already in place (8).

In this paper we argue that effective program management should be fundamentally based on first strengthening the manual and paper based systems while considering also systems that are tacit and not pronounced without which useful computer based HIS cannot be deployed . The empirical basis of the argument comes from the analysis of two kinds of information systems - manual and tacit - related to immunization program for measles in Mozambique. We examine both

${ }^{1}$ Department of Immunology, Instituto Nacional de Saude, Maputo, Mozambique; ${ }^{2}$ Department of General Practice and Community Medicine, University of Oslo, Norway; ${ }^{3}$ Department of Informatics, University of Oslo, Oslo, Norway 
systems, comparing the data generated in order to look at any existing discrepancies.

EPI information flow in Mozambique: EPI is a national program coordinated and controlled by the Ministry of Health. It provides services through all health centers at fixed and mobile post vaccinations, targeting populations living in the catchment area, including those that are hard to reach. Data for the EPI information system are generated at the initial contact between a child and the health facility (HF). The information is recorded in two pre coded immunization tally sheets - one records BCG, 3 doses of DTP and hepatitis B, polio and measles vaccines, and the second tally sheet records tetanus vaccine. At the first vaccination to a child the mother is provided with the Road to Health Card (RH Card) where all the vaccinations, growth monitoring and disease treatment during the child's first five years of life are registered. The mother keeps the RH Card and she is requested by the health staff to bring it on every occasion that her child seeks any health attention at the health facility. The health facility does not keep any individual based information regarding vaccination.

The RH Card constitutes the basic data source of the immunization status of children in the area. On a monthly basis at the health facilities, the registers of the number of each vaccine doses administered are colleted in a single form and sent to the district level. The data are aggregated at the district level into a district form, which does not discriminate information by health facilities. The district form is sent to the provincial level on a monthly basis where the data are entered into a computer database. Provincial reports are sent to the national level on a quarterly basis. This information is used as the numerator to calculate the indicator of vaccine coverage. The denominator is the catchment's population information obtained from the census (collected once in a decade).

Empirical approach: The study was carried out in Manica Province that had a population of $1,200,000$ inhabitants in 2002 (9). The province is located in the central region of Mozambique and has nine districts. The health data flows from the health facility to the district where the data are compiled and sent to the province, and from there to national level. Measles vaccination coverage is reported to be around 92\%, and in 2002 the coverage reached $101 \%$. Despite the high vaccine coverage, viral circulation of measles continues with sporadic cases reported every year (10).

Due to the centralized system of EPI management, the province suffered from frequent shortages from RH Card due to the infrequent and insufficient supply of materials from the central level in the previous five years. As the result of these shortages a large component of this information system currently relies on verbal or unsaid information. However, the Manica Provincial Health Directorate tried to minimize the problem of lack of $\mathrm{RH}$ Cards by recording the weight and the vaccination status on a simple paper sheet, which was given to the mother. During the next visit to the health-facility, the information was recorded in the $\mathrm{RH}$ Card if it was available at that time.

In 2002, Manica province was facing a measles epidemic (10) and local authorities inquired to assess vaccination coverage and to know the efficacy of the measles vaccine. However, due to the lack of individual vaccine registries at the health facility, the only available source of individual-level information was the $\mathrm{RH}$ Card in possession of the mothers. The above-referred research analyzed the measles surveillance system and showed that it was not valid and capable of assessing indicators like vaccine efficacy. The present study was designed to evaluate the accuracy of the mothers' verbal information regarding measles vaccination status as narrated to the health workers. The results of this study were expected to help the health worker to decide whether or not to use the mother vaccination information in the absence of the $\mathrm{RH}$ Card to decide whether or not to vaccinate the child. The decision makers at the province and central levels were also expected to use the same information to calculate measles vaccine efficacy by calculating the disease attack rate among vaccinated and non-vaccinated children as well as measles vaccine coverage among children age 1035 months of age.

Previous studies have evaluated the accuracy of a mother's information in different contexts; however, the use of verbal information to calculate vaccine coverage has been widely explored but remains controversial (2, 11-15). Some studies reported errors of overestimation in recalling measles vaccine (15); others reported mothers' underestimation of their child's vaccination status $(12,13)$. Nevertheless, there are also studies that reported accurate assessment of child vaccination status by their mothers $(11,14)$. This paper's aim is to describe the accuracy of mothers' information regarding the measles vaccination status of their children (people-based information source) compared with information recorded in the $\mathrm{RH}$ card (manual information source). This information will help determine the accuracy of each information system and validate its use for calculating vaccine efficacy and coverage.

\section{Methods}

A cross sectional study was conducted during six weeks between November and December of 2002. The sample size for the study was calculated with the Epi start program. Using a 95\% level of confidence and assuming $80 \%$ of the children having a complete vaccination status with a 3\% margin of error and 15\% contingency, a total of 840 subjects were included in the study. 
The study was carried out in two health centers in the Manica Province: one in Chimoio City and one in Gondola District. The health centers were chosen due to easy accessibility for the researcher and the relatively high flow of clients seeking immunization services in these facilities.

The data for this study were obtained from two sources: RH Cards and mothers' verbal information.

Every third mother with a child of 10 to 35 months age, coming to the health-facility for any reason, whether or not she had a RH Card, was invited to participate in the study. The targeted age group was chosen to reduce recall bias. The mother's verbal consent was asked to include her in the study. Mothers were also asked about their background, reproductive history and education levels and their child's measles vaccination status. After the interview, mothers were asked to present their child's RH Card for inspection, and at that moment they were divided into two groups: mothers with the $\mathrm{RH}$ card (Group A) and mothers without the RH card (Group B).

Data were collected in both individual and anonymous forms. Interviewers were trained and supervised by one of the authors of this paper on a weekly basis, for the duration of the study. Quality control of data was conducted by a double check method of a random sample of $10 \%$ of questionnaires. All randomized questionnaires were given to one of the authors to verify the data by visiting respective households. Finally, the data entered in Epi Info 6.0 database (16) was reviewed and cleaned before data were analyzed.

The background information collected in the interviews included various details about the mother such as age, obstetric history (number of gestations, parity and abortions), schooling, civil status, as well as information regarding the status of their children's measles vaccination.

The baseline summary statistics were computed as means for continuous variables and as proportions for categorical variables. Means were compared using t-tests and the statistical difference in the proportions was compared using chi-square test with a $5 \%$ significance level.

Comparative analysis of proportions was conducted on the following information:

(a) Mothers' information regarding their children's measles vaccination status

(b) Information recorded in their RH Cards (group A)

(c) Information from mothers with and without the $\mathrm{RH}$

Cards regarding their children's measles vaccination status (group A versus group B).
The term "mothers without RH Cards" included mothers who had lost or forgotten to bring the RH Cards in addition to those who never received a RH Card. The analysis sought to examine the validity of mothers' verbal information regarding measles vaccination status of their children when compared with the RH Card records; the information on the RH Cards was defined by us as the "gold standard". We measured the degree of accuracy of information regarding measles vaccination in respective children by the proportion of mothers verbally reporting information similar to that recorded in the RH Card. For those mothers that had the RH Card (validated sample), we calculated the positive predictive value as a percentage of children reported to be immunized against measles and cross checked with information documented in the RH Cards. The negative predictive value was calculated as the percentage of children reported as not immunized confirmed by the RH Card record. After comparison of mothers' verbal information with the "gold standard" RH Card, we compared the verbal information of those mothers with those that had not presented $\mathrm{RH}$ Cards by using the differences in the two proportions. We also compared the reproductive history and education characteristics of these two groups.

We calculated recall bias for those mothers that presented the RH Card by calculating the proportion of those mothers that responded with information about their child's vaccination status that was incongruous with the information on the RH Card.

\section{Results}

Of a total of 840 mothers that were interviewed, $64.3 \%$ presented a RH Card. Of the 540 mothers with RH Cards, $79.3 \%$ were interviewed and their responses regarding their child's measles vaccine status were compared to the gold standard to test for accuracy (see Table 1).

Of 447 children with a history of measles vaccine reported by their mothers, 95.8\% had measles vaccination recorded in the RH Card. Of 93 children with a negative history of measles vaccine reported by their mothers, $82 \%$ of 93 had no measles vaccination recorded on the RH Card. The sensitivity and specificity of reported measles vaccination were $95.7 \%$ (95\%CI 93.8-97.6\%) and $80.0 \% \quad(95 \%$ CI $\quad 71.9-88.0 \%)$, respectively.

Only 17 (3.8\%) of mothers whose children had records of their vaccination in their RH Cards (445) had recall bias for the negative event (child not being vaccinated). Of the mothers whose children had no record of a vaccination in their RH Cards (95), 19 (20.0\%) had recall bias for the positive event (child being vaccinated). 
Table 1: Comparison of children's measles vaccination status as reported verbally by mothers and as recorded on the Road to Health Cards

\begin{tabular}{|c|c|c|c|}
\hline \multirow[b]{2}{*}{ Mothers information } & \multicolumn{2}{|c|}{ Information from RH Card } & \multirow[b]{2}{*}{ Total } \\
\hline & Vaccinated children & Non vaccinated children & \\
\hline Vaccinated children & 428 & 19 & 447 \\
\hline Non vaccinated children & 17 & 76 & 93 \\
\hline Total & 445 & 95 & 540 \\
\hline
\end{tabular}

Comparison of patterns of mother's information regarding measles vaccine among those with and without cards: The pattern of answers (see Table 2) of mothers who had not presented the RH Card were $10 \%$ higher $(\mathrm{P}<0.001)$ than those with cards with respect to affirmative answers that the children had received measles vaccination. In the sample of mothers without the RH Card, only $2.0 \%$ had not received a RH Card,
$54.0 \%$ had forgotten to bring it, and the remaining $44.0 \%$ had lost it. The baseline characteristics of this sample (see Table 2), discloses some of the differences between the two groups, with respect to the status of their schooling and the frequency of abortions. There was no statistical difference in the mean age of the mothers with and without the RH Card for their children. Furthermore, $81.0 \%$ of the children were under 24 months of age.

Table 2: General characteristics of mothers with and without the Road to Health Card

\begin{tabular}{|c|c|c|c|}
\hline Characteristics & $\begin{array}{l}\text { Mothers with } \\
\text { Road to Health Card } \\
(\mathrm{N}=540)\end{array}$ & $\begin{array}{l}\text { Mothers without } \\
\text { Road to Health Card } \\
(\mathrm{N}=300)\end{array}$ & P-Value \\
\hline Average age (years) & 24.24 & 24.59 & 0.81 \\
\hline Average children age Obstetric history (average value) & 17.74 & 18.08 & 0.5 \\
\hline Pregnancies & 3.23 & 2.95 & 0.06 \\
\hline Abortions & 0.28 & 0.14 & $<0.001$ \\
\hline Deliveries & 2.72 & 2.71 & 0.94 \\
\hline Average schooling & 4.6 & 3.61 & $<0.001$ \\
\hline Civil status, married (\%) & 82.9 & 93 & $<0.001$ \\
\hline History of measles vaccination (\%) & 82 & 92 & $<0.001$ \\
\hline
\end{tabular}

\section{Discussion}

This study addresses the accuracy of mothers' information regarding measles vaccination. Since the childhood vaccination program (EPI) includes seven different vaccines with varying timings, one of the limitations of the study is that our results are valid for one vaccine. Clearly, the results provide no information about the adequacy and timeliness of vaccination. This information could be epidemiologically important. Recall bias increases with the age of children and by more detailed information requested such as vaccination date, number of doses received) (17), and the type of vaccine (13).

The measles vaccine administrated at 9 months of age i.e. at the end of the childhood immunization program, is more likely to be recalled by the mother. Therefore, we believe it could be assessed easily with a relatively small recall bias. To further decrease the recall bias, an age group 10-35 months was chosen. However, a limitation of the study is that we cannot generalize the results for other EPI vaccines. The results of this study show that children between 10-35 months of age vaccinated against measles were recognized as being vaccinated by almost all mothers (Se=95.7\%; +/ 1, 87). We considered this as showing high sensitivity compared with a similar study in Sudan where the reported sensitivity of mothers' reports is $87.1 \%$ among children between 11 and 16 months of age (11).

The veracity of the mothers' verbal information in the case of the non-vaccinated children was lower (Sp $80.0 \%$; +/- 7,23). This indicates that $20.0 \%$ of the mothers had a recall bias for a positive event that did not occur. Similar results were found in Sudan (11). In part, this result may be due to such factors as the low numbers of mothers with a RH Card and children without measles vaccine; difficulties in recalling with reliability the event that did not occur (not being vaccinated); and a larger propensity to avoid information of negative events (no vaccination). The information of the mothers without a RH Card apparently similar to that provided by mothers with RH Card, the data show stronger propensity to affirmatively report the measles vaccination status of their children (Table 2).

Despite the difficult situation of shortage of RH Cards, the majority of mothers classified as "mothers without 
RH Card" had previously received a RH Card, although a large percentage of them either lost or forgotten the card at home. This indicates that the distribution of cards needs to be accompanied with educational messages stressing the importance of safe guarding the cards. In addition, it also fundamentally indicates the need to create an individual record system that is maintained at the health facility level.

The accuracy of the reports made by mothers with RH Cards cannot be used as a sole guide to interpret the information provided by mothers without these cards. Program managers and individual health workers should be more concerned about the problem of over-reporting rather than under-reporting of measles vaccination. If the interventions are based solely on the mothers' information, the overestimation of vaccine coverage and underestimation of vaccine efficacy can be expected. This finding is supported by another study that concludes that the method of obtaining immunization status through recall survey is not sensitive enough for accurate estimation of vaccine coverage (18). The reproductive history and level of education differences between these two groups of mothers can also partially explain the differences in the patterns of answers obtained. Responses from less educated, married women with larger family responsibilities, were statistically significant amongst the group of mothers without the $\mathrm{RH}$ Card, and might be related with the high numbers of cards lost or forgotten at home. A study in Guatemala showed similar associations and the authors there argue that more educated women may be more health conscious, and therefore may have obtained the vaccination and retained theirs children's RH Card (17). Nevertheless, factors such as the age of the mother, previous gestations, number of children and the age of the last child showed similar characteristics among two groups.

Interestingly, the higher frequency of abortions among the group of mothers without RH Card was an important finding. However, without knowing the reasons behind the abortions, it was not possible to further explore this issue.

Our discussion of verbal and documented information relating to child immunization provides some implications for the design and implementation of information systems, either manual or computer based, relating to the history of immunization. Firstly, given the high percentage of cards that are lost or forgotten at home, it becomes urgent to develop a mother-specific system with the child's immunization details stored at the health facility. Such a system could be either paper or computer based, or some appropriate combination of the two depending on the local context. Secondly, the higher percentage of affirmative answers given by mothers without cards is indicative of the possibility of getting incorrect information. To ensure higher quality of data, it is necessary to develop greater validation mechanisms for such cases. Thirdly, and related to the second conclusion, there are needs for education campaigns to emphasize the importance of keeping and bringing the RH Cards to the health facility every time that the child seeks health care.

\section{Acknowledgments}

We thank Dr. Francisco Mbofana for the support during data entry. We gratefully acknowledge the financial support from Integrated Health Project in Manica Province in 2002. We also thank Dr. Caroline De Schacht for suggestions and comments on the final draft.

\section{References}

1. Measles eradiation recommendations from a meeting cosponsored by the World Health Organization, the Pan American Health Organization, and CDC. MMWR Recomm Rep. 1997; 46(RR-11): 1-20.

2. Expanded programme on immunization (EPI). Lot Quality Assurance survey to assess immunization coverage. Wkly Epidemiol Rec. 1995;70(37):261-4.

3. Mavimbe JC, Braa J, Bjune G. Assessing immunization data quality from routine reports in Mozambique. BMC Public Health 2005; 5:108.

4. Salazar A. Challenges to Information Systems Implementation and Organisational Change Management: Insights from the Health Sector in Ecuador. EJSDC. 2004; 16(6): 1-16.

5. Puri S, Byrne E, Nhampossa J, Quraishi Z. Contextuality of Participation in IS Design: A Developing Country Perspective. In proceedings I, editor. Artful Integration: Interweaving media, materials and practices; 2004; Toronto, Canada.

6. Braa J, Monteiro E, Sahay s. Network for action MIS quarterly. 2004; 28(3): 337-62.

7. Lippeveld T, Sauborn R, Boclert C. Design and implementation of Health Information Systems. Washington DC: World Health Organisation; 2000.

8. Raghvendar c, Sahay S. Computer-based health information systems - Projects for computerization or health management? Empirical experiences from India to be published in the book titled Information communication technologies and human development: Opportunities and challenges by Idea group 1006.

9. INS Projeccao Anual da Populacao por Distritos 1997-2010. In: Statistic, editor.

10. Jani JV, Jani IV, Araujo C, Sahay S, Barreto J, Bjune G. Assessment of routine surveillance data as a tool to investigate measles outbreaks in Mozambique. BMC Infect Dis. 2006; 6:29.

11. Gareaballah ET, Loevinsohn BP. The accuracy of mother's reports about their children's vaccination 
status. Bull World Health Organ.1989; 67(6): 66974.

12. Valadez JJ, Weld LH. Maternal recall error of child vaccination status in a developing nation. Am J public Health. 199; 82(1): 120-2.

13. Suarez L, Simpson DM, Smith DR. Errors and correlates in parental recall of child immunizations: effects on vaccination coverage estimates. Pediatrics. 1997; 99(5): e3.

14. Langsten R, Hill K. The accuracy of mothers' reports of child vaccination: evidence from rural Egypt. Soc sci med. 1998; 46(9): 1205-12.

15. Hawe P, Wilson A, Fahey P, Field P, Commingham AL, Baker M, et al. The validity of parental report of vaccination as a measure of a child's measles immunisation status. Med J Aust. 1991; 155(10): 681,4-6.
16. AG Dean JD, D Coulombier, AH Burton, KA Brendel, DC Smith, et al. Epi Info: A world processing, database, and statistics program for public health on IBM-compatible microcomputers. 6.0 ed. Atlanta (GA): Center for Disease Control and Prevention; 1995.

17. Goldman N, Pebley AR. Health cards, maternal reports and the measurement of immunization coverage: the example of Guatemala. Soc Sci Med 1994;38(8):1075-89.

18. Ramakrishnan R, Rao TV, Sundaramoorthy l, Johua V. Magnitude of recall bias in the estimation of immunization coverage and its determinants. Indian Pediatr. 1999; 36(9): 881-5. 